\title{
OPTIMAL WEAK TYPE ESTIMATES FOR DYADIC-LIKE MAXIMAL OPERATORS
}

\author{
Eleftherios N. Nikolidakis \\ University of Crete, Department of Mathematics \\ Heraklion 71409, Crete, Greece; lefteris@math.uoc.gr
}

\begin{abstract}
We provide sharp weak estimates for the distribution function of $\mathcal{M} \phi$ when on $\phi$ we impose $L^{1}, L^{q}$ and $L^{p, \infty}$ restrictions. Here $\mathcal{M}$ is the dyadic maximal operator associated to a tree $\mathcal{T}$ on a non-atomic probability measure space. As a consequence we produce that the inequality $\left\|\mathcal{M}_{\mathcal{T}} \phi\right\|_{p, \infty} \leq\|\| \phi \|_{p, \infty}$ is sharp allowing every possible value for the $L^{1}$ and the $L^{q}$ norm for a fixed $q$ such that $1<q<p$, where $\|\mid \cdot\| \|_{p, \infty}$ is the integral norm on and $\|\cdot\|_{p, \infty}$ the usual quasi norm on $L^{p, \infty}$.
\end{abstract}

\section{Introduction}

The dyadic maximal operator on $\mathbf{R}^{n}$ is defined by

$$
\mathcal{M}_{d} \phi(x)=\sup \left\{\frac{1}{|Q|} \int_{Q}|\phi(u)| d u: x \in Q, Q \subseteq \mathbf{R}^{n} \text { is a dyadic cube }\right\}
$$

for every $\phi \in L_{\text {loc }}^{1}\left(\mathbf{R}^{n}\right)$ where the dyadic cubes are those formed by the grids $2^{-N} \mathbf{Z}^{n}$ for $N=1,2, \ldots$ and $|A|$ is the Lesbesgue measure of any measurable subset $A$ of $R^{n}$. It is easy to prove by using the definition of $\mathcal{M}_{d}$ that it satisfies the following weak type $(1,1)$ inequality

$$
\left|\left\{x \in \mathbf{R}^{n}: \mathcal{M}_{d} \phi(x) \geq \lambda\right\}\right| \leq \frac{1}{\lambda} \int_{\left\{\mathcal{M}_{d} \phi \geq \lambda\right\}}|\phi(u)| d u
$$

for every $\phi \in L^{1}\left(\mathbf{R}^{n}\right)$ and every $\lambda>0$. Tis inequality is sharp as can be easily seen by considering characteristic functions over dyadic cubes. Using the fact that

$$
\left\|\mathcal{M}_{d} \phi\right\|_{p}^{p}=\int_{0}^{\infty} p \lambda^{p-1}\left|\left\{\mathcal{M}_{d} \phi \geq \lambda\right\}\right| d \lambda
$$

and in the sequel inequality (1.2) along with Fubini's theorem we easily get the following $L^{p}$ inequality known as Doob's inequality

$$
\left\|\mathcal{M}_{d} \phi\right\|_{p} \leq \frac{p}{p-1}\|\phi\|_{p}
$$

for every $p>1$ and every $\phi \in L^{p}\left(\mathbf{R}^{n}\right)$, which is proved to be best possible (see [2,3] for the general martingales and [10] for the dyadic ones).

A way of studying the dyadic maximal operator is the introduction of the so called Bellman functions (see [8]). Actually, we define for every $p>1$

$$
B_{p}(f, F)=\sup \left\{\frac{1}{|Q|} \int_{Q}\left(\mathcal{M}_{d} \phi\right)^{p}: \frac{1}{|Q|} \int_{Q} \phi^{p}=F, \frac{1}{|Q|} \int_{Q} \phi=f\right\}
$$

doi:10.5186/aasfm.2013.3817

2010 Mathematics Subject Classification: Primary 47A30.

Key words: Dyadic, maximal. 
where $Q$ is a fixed dyadic cube, $\phi$ is nonnegative in $L^{p}(Q)$ and $f, F$ are such that $0<f^{p} \leq F . B_{p}(f, F)$ has been computed in [5]. In fact it has been shown that $B_{p}(f, F)=F \omega_{p}\left(f^{p} / F\right)^{p}$ where $\omega_{p}:[0,1] \rightarrow\left[1, \frac{p}{p-1}\right]$ is the inverse function of

$$
H_{p}(z)=-(p-1) z^{p}+p z^{p-1} .
$$

This has been proved in a much more general setting of tree like maximal operators on non-atomic probability spaces. The result turns out to be independent of the choice of the measure space. The study of these operators has been continued in [7] where the Bellman functions of them in the case $p<1$ have been computed. As in [5] and [7] we will follow the moregeneral approach. So for a tree $\mathcal{T}$ on a non atomic probability measure space $(X, \mu)$, we define the associated dyadic maximal operator, namely

for every $\phi \in L^{1}(X, \mu)$.

$$
\mathcal{M}_{\mathcal{T}} \phi(x)=\sup \left\{\frac{1}{\mu(I)} \int_{I}|\phi| d \mu: x \in I \in \mathcal{T}\right\}
$$

As it can be seen in [9], $\mathcal{M}_{\mathcal{T}}: L^{p, \infty} \rightarrow L^{p, \infty}$ is a continuous operator and satisfies the following inequality

$$
\left\|\mathcal{M}_{\mathcal{T}} \phi\right\|_{p, \infty} \leq\|\| \phi \|_{p, \infty}
$$

where $\|\cdot\|_{p, \infty}$ is the usual quasi-norm on $L^{p, \infty}$ defined by

$$
\|\phi\|_{p, \infty}=\sup \left\{\lambda \mu(\{\phi \geq \lambda\})^{1 / p}: \lambda>0\right\} .
$$

and $\mid\|\cdot\| \|_{p, \infty}$ is the integral norm on $L^{p, \infty}$ given by $\|\phi \mid\|_{p, \infty}=\sup \left\{\mu(E)^{-1+\frac{1}{p}} \int_{E}|\phi| d \mu: E\right.$ measurable subset of $X$ such that $\left.\mu(E)>0\right\}$. $\||\cdot|\|_{p, \infty}$ and $\|\cdot\|_{p, \infty}$ are equivalent because of the following

$$
\|\phi\|_{p, \infty} \leq\|\| \phi\left\|_{p, \infty} \leq \frac{p}{p-1}\right\| \phi \|_{p, \infty}, \forall \phi \in L^{p, \infty},
$$

which can be seen in [4]. In this paper we prove that inequality (1.5) is sharp and independent of the $L^{1}$ and $L^{q}$ norm of $\phi$, for a fixed $q$ such that $1<q<p$. In fact we prove a stronger result, by evaluating the following function of $\lambda>0$

$$
\begin{aligned}
& S(f, A, F, \lambda) \\
& =\sup \left\{\mu\left(\left\{\mathcal{M}_{\mathcal{T}} \phi \geq \lambda\right\}\right): \phi \geq 0, \int_{X} \phi d \mu=f, \int_{X} \phi^{q} d \mu=A,\|\mid \phi\|_{p, \infty}=F\right\},
\end{aligned}
$$

where $(f, A, F)$ is on the domain of the extremal problem. That is we prove the following

Theorem 1.1. For $f$, $A$ such that $f^{q}<A \leq \Gamma f^{p-q / p-1} F^{p(q-1) / p-1}$ and $0<f \leq$ $F$ the following hold

$$
S(f, A, F, \lambda)=\min \left\{1, G_{f, A}(\lambda), \frac{F^{p}}{\lambda^{p}}\right\}
$$

where

$$
G_{f, A}(\lambda)=\sup \left\{\mu\left(\left\{\mathcal{M}_{\mathcal{T}} \phi \geq \lambda\right\}\right): \phi \geq 0, \int_{X} \phi d \mu=f, \int_{X} \phi^{q} d \mu=A\right\}
$$


In fact, $G_{f, A}(\lambda)$ has been precisely computed in [6] by using sharp inequalities on a certain class of functions which is enough to describe the related problem. In this paper we avoid the technique used in [6] and refine this result by proving the theorem mentioned using a different approach. As a corrolary we obtain the following

Corollary 1.1. The following is true

$$
\sup \left\{\left\|\mathcal{M}_{\mathcal{T}} \phi\right\|_{p, \infty}: \phi \geq 0, \int_{X} \phi d \mu=f, \int_{X} \phi^{q} d \mu=A,\|\| \phi \|_{p, \infty}=F\right\}=F,
$$

that is, (1.5) is sharp allowing every value of the integral and the $L^{q}$-norm of $\phi$.

This paper is organized as follows: In Section 2 we provide some lemmas and facts concerning non-atomic probability measure spaces and trees on them. In Section 3 we find the domain of the extremal problem for the case $F=1$. This is done by finding sharp inequalities relating the $L^{1}$ and $L^{q}$ norm of a measurable function $\phi$ under the weak condition $\||\phi|\|_{p, \infty}=1$. Krein-Milman theorem is a tool for us in order to find these sharp inequalities. At last in section 4 we precisely evaluate $S(f, A, 1, \lambda)$. We need also to mention that all the estimates are independent of the measure space $(X, \mu)$ and the tree $\mathcal{T}$.

\section{Preliminaries}

Let $(X, \mu)$ be a non-atomic probability measure space. We state the following lemma which can be found in [1].

Lemma 2.1. Let $\phi:(X, \mu) \rightarrow \mathbf{R}^{+}$and $\phi^{*}$ the decreasing rearrangement of $\phi$, defined on $[0,1]$. Then

$$
\int_{0}^{t} \phi^{*}(u) d u=\sup \left\{\int_{E} \phi d \mu: E \text { measurable subset of } X \text { with } \mu(E)=t\right\}
$$

for every $t \in[0,1]$, with the supremum attained.

We prove now the following

Lemma 2.2. Let $\phi: X \rightarrow \mathbf{R}^{+}$be measurable and $I \subseteq X$ be measurable with $\mu(I)>0$. Suppose that $\frac{1}{\mu(I)} \int_{I} \phi d \mu=s$. Then for every $t$ such that $0<t \leq \mu(I)$ there exists a measurable set $E_{t} \subseteq I$ with $\mu\left(E_{t}\right)=t$ and $\frac{1}{\mu\left(E_{t}\right)} \int_{E_{t}} \phi d \mu=s$.

Proof. Consider the measure space $(I, \mu / I)$ and let $\psi: I \rightarrow \mathbf{R}^{+}$be the restriction of $\phi$ on $I$ that is $\psi=\phi / I$. Then, if $\psi^{*}:[0, \mu(I)] \rightarrow \mathbf{R}^{+}$is the decreasing rearrangement of $\psi$, we have that

$$
\frac{1}{t} \int_{0}^{t} \psi^{*}(u) d u \geq \frac{1}{\mu(I)} \int_{0}^{\mu(I)} \psi^{*}(u) d u=s \geq \frac{1}{t} \int_{\mu(I)-t}^{\mu(I)} \psi^{*}(u) d u .
$$

Since $\psi^{*}$ is decreasing, we get the inequalities in (2.1), while the equality is obvious since

$$
\int_{0}^{\mu(I)} \psi^{*}(u) d u=\int_{I} \phi d \mu .
$$

From (2.1) it is easily seen that there exists $r \geq 0$ such that $t+r \leq \mu(I)$ with

$$
\frac{1}{t} \int_{r}^{t+r} \psi^{*}(u) d u=s
$$


It is also easily seen that there exists $E_{t}$ measurable subset of $I$ such that

$$
\mu\left(E_{t}\right)=t \quad \text { and } \quad \int_{E_{t}} \phi d \mu=\int_{r}^{t+r} \psi^{*}(u) d u
$$

since $(X, \mu)$ is non-atomic. From (2.2) and (2.3) we get the conclusion of the lemma.

We now call two measurable subsets of $X$ almost disjoint if $\mu(A \cap B)=0$. We give now the following

Definition 2.1. A set $\mathcal{T}$ of measurable subsets of $X$ will be called a tree if the following conditions are satisfied:

(i) $X \in \mathcal{T}$ and for every $I \in \mathcal{T}$ we have that $\mu(I)>0$.

(ii) For every $I \in \mathcal{T}$ there corresponds a finite or countable subset $C(I) \subseteq \mathcal{T}$ containing at least two elements such that

(a) the elements of $C(I)$ are pairwise almost disjoint subsets of $I$,

(b) $I=\cup C(I)$.

(iii) $\mathcal{T}=\bigcup_{m \geq 0} \mathcal{T}_{(m)}$ where $\mathcal{T}_{0}=\{X\}$ and $\mathcal{T}_{(m+1)}=\bigcup_{I \in \mathcal{T}_{(m)}} C(I)$.

(iv) $\lim _{m \rightarrow+\infty} \sup _{I \in \mathcal{T}_{(m)}} \mu(I)=0$.

From [5] we get the following

Lemma 2.3. For every $I \in \mathcal{T}$ and every $\alpha$ such that $0<\alpha<1$ there exists a subfamily $\mathcal{F}(I) \subseteq \mathcal{T}$ consisting of pairwise almost disjoint subsets of $I$ such that

$$
\mu\left(\bigcup_{J \in \mathcal{F}(I)} J\right)=\sum_{J \in \mathcal{F}(I)} \mu(J)=(1-\alpha) \mu(I) .
$$

Let now $(X, \mu)$ be a non-atomic probability measure space and $\mathcal{T}$ a tree as in Definition 1.1. We define the associated maximal operator to the tree $\mathcal{T}$ as follows: For every $\phi \in L^{1}(X, \mu)$ and $x \in X$, then

$$
\mathcal{M}_{\mathcal{T}} \phi(x)=\sup \left\{\frac{1}{\mu(I)} \int_{I}|\phi| d \mu: x \in I \in \mathcal{T}\right\} .
$$

\section{The domain of the extremal problem}

Our aim is to find the exact allowable values of $(f, A, F)$ for which there exists $\phi:(X, \mu) \rightarrow \mathbf{R}^{+}$measurable such that

$$
\int_{X} \phi d \mu=f, \quad \int_{X} \phi^{q} d \mu=A \quad \text { and } \quad\|\phi\|_{p, \infty}=F .
$$

We find it in the case where $F=1$. For the beginning assume that $(f, A)$ are such that there exist $\phi$ as in (3.1). We set $g=\phi^{*}:[0,1] \rightarrow \mathbf{R}^{+}$. Then

$$
\int_{0}^{1} g=f, \quad \int_{0}^{1} g^{q}=A \quad \text { and } \quad\|\| g \|_{p, \infty}^{[0,1]}=1
$$

where

$\||| g \mid\|_{p, \infty}^{[0,1]}=\sup \left\{|E|^{-1+\frac{1}{p}} \int_{E} g: E \subset[0,1]\right.$ Lebesque measurable such that $\left.|E|>0\right\}$. 
This is true because of the definition of the decreasing rearrangement of $\phi$ and Lemma 2.1. In fact since $g$ is decreasing $\||| g \mid\|_{p, \infty}$ is equal to

$$
\sup \left\{t^{-1+\frac{1}{p}} \int_{0}^{t} g: 0<t \leq 1\right\} .
$$

Of course, we should have that $0<f \leq 1$ and $f^{q} \leq A$. We give now the following

Definition 3.1. If $n \in \mathbf{N}$, and $h:[0,1) \rightarrow \mathbf{R}^{+}, h$ will be called $\frac{1}{2^{n}}$-step if it is constant on each interval

$$
\left[\frac{i-1}{2^{n}}, \frac{i}{2^{n}}\right), \quad i=1,2, \ldots, 2^{n} .
$$

Now for $n \in \mathbf{N}$ and $0<f \leq 1$ fixed, we set

$$
\Delta_{n}(f)=\left\{h:[0,1] \rightarrow \mathbf{R}^{+}: h \text { is a } \frac{1}{2^{n}} \text {-step function, } \int_{0}^{1} h=f,\|\| h \|_{p, \infty}^{[0,1]} \leq 1\right\} .
$$

Then

$$
\Delta_{n}=\Delta_{n}(f) \subset L^{p, \infty}([0,1])
$$

where we use the \|\|$\cdot \|\left.\right|_{p, \infty} ^{[0,1]}$ norm for functions defined on $[0,1] . \Delta_{n}$ is also convex, that is,

$$
h_{1}, h_{2} \in \Delta_{n} \Longrightarrow \frac{h_{1}+h_{2}}{2} \in \Delta_{n} .
$$

Additionally, we have the following

Lemma 3.1. $\Delta_{n}$ is compact subset of $L^{p, \infty}([0,1])=Y$ where the topology on $Y$ is that endowed by \|\|$\cdot\|\|_{p, \infty}^{[0,1]}$.

Proof. $\left(Y,|||\cdot| \|_{p, \infty}\right)$ is a Banach space. So, especially a metric space. As a consequence we just need to prove that $\Delta_{n}$ is sequentially compact.Let now $\left(h_{i}\right)_{i} \subset$ $\Delta_{n}$. It is now easy to see by a finite diagonal argument that there exists $\left(h_{i_{j}}\right)_{j}$ subsequence and $h:[0,1] \rightarrow \mathbf{R}^{+}$. such that $h_{i_{j}} \rightarrow h$ uniformly on $[0,1]$. Then obviously $\int_{0}^{1} h=f,\||| h \mid\|_{p, \infty}^{[0,1]} \leq 1$, so $h \in \Delta_{n}$. Additionally

$$
\begin{aligned}
\left\|\left|h_{i_{j}}-h\right|\right\|_{p, \infty}^{[0,1]} & =\sup \left\{|E|^{-1+\frac{1}{p}} \int_{E}\left|h_{i_{j}}-h\right|:|E|>0\right\} \\
& \leq \sup \left\{\left|\left(h_{i_{j}}-h\right)(t)\right|, t \in[0,1]\right\} \rightarrow 0
\end{aligned}
$$

as $j \rightarrow \infty$. That is $h_{i_{j}} \stackrel{Y}{\longrightarrow} h \in \Delta_{n}$. Consequently, $\Delta_{n}$ is a compact subset of $L^{p, \infty}([0,1])$.

We give now the following known

Definition 3.2. For a closed convex subset $K$ of a topological vector space $Y$, and for a $y \in K$ we say that $y$ is an extreme point of $K$, if whenever $y=\frac{x+z}{2}$, with $x, z \in K$ it is implied that $y=x=z$. We write $y \in \operatorname{ext}(K)$.

Definition 3.3. For a subset $A$ of a topological vector space $Y$ we set

$$
\operatorname{conv}(A)=\left\{\sum_{i=1}^{n} \lambda_{i} x_{i}: \lambda_{i} \geq 0, x_{i} \in A, n \in \mathbf{N}^{*}, \sum_{i=1}^{n} \lambda_{i}=1\right\} .
$$

We call $\operatorname{conv}(A)$ the convex hull of $A$. 
We state now the following well known

Theorem 3.1. (Krein-Milman) Let $K$ be a convex, compact subset of a locally convex topological vector space $Y$. Then $K=\overline{\operatorname{conv}(\operatorname{ext}(K))}$, that is, $K$ is the closed convex hull of its extreme points.

According now to Lemma 3.1 we have that

$$
\Delta_{n}=\overline{\operatorname{conv}\left[\operatorname{ext}\left(\Delta_{n}\right)\right]}{ }^{L^{p, \infty}([0,1])} .
$$

We find now the set $\operatorname{ext}\left(\Delta_{n}\right)$.

Lemma 3.2. Let $g \in \operatorname{ext}\left(\Delta_{n}\right)$. Then for every $i \in\left\{1,2, \ldots, 2^{n}\right\}$ such that $\left(\frac{i}{2^{n}}\right)^{1-\frac{1}{p}} \leq f$, we have that

$$
\sup \left\{|E|^{-1+\frac{1}{p}} \int_{E} g:|E|=\frac{i}{2^{n}}\right\}=1 .
$$

Proof. We prove it first when $i=1$ and $\left(\frac{1}{2^{n}}\right)^{1-\frac{1}{p}} \leq f$. It is now easy to see that $g \in \operatorname{ext}\left(\Delta_{n}\right)$ if and only if $g^{*} \in \operatorname{ext}\left(\Delta_{n}\right)$. So we just need to prove that $\int_{0}^{1 / 2^{n}} g^{*}=\left(\frac{1}{2^{n}}\right)^{1-\frac{1}{p}}$. We write

$$
g^{*}=\sum_{i=1}^{2^{n}} \alpha_{i} \xi_{I_{i}} \text { with } I_{i}=\left[\frac{i-1}{2^{n}}, \frac{i}{2^{n}}\right)
$$

and $\alpha_{i} \geq \alpha_{i+1}$ for every $i \in\left\{1,2, \ldots, 2^{n}-1\right\}$. Suppose now that $\alpha_{1}<2^{n / p}$, and that $\alpha_{1}>\alpha_{2}$ (the case $\alpha_{1}=\alpha_{2}$ is handled in an analogous way). For a suitable $\varepsilon>0$ we set

$$
\left.g_{1}=\sum_{i=1}^{2^{n}} \alpha_{i}^{(1)} \xi_{I_{i}}, \quad g_{2}=\sum_{i=1}^{2^{n}} \alpha_{i}^{(2)} \xi_{I_{i}}, \quad \text { where } \begin{array}{ll}
\alpha_{1}^{(1)}=\alpha_{1}+\varepsilon, & \alpha_{2}^{(1)}=\alpha_{2}-\varepsilon \\
\alpha_{1}^{(2)}=\alpha_{1}-\varepsilon, & \alpha_{2}^{(2)}=\alpha_{2}+\varepsilon
\end{array}\right\}
$$

and $\alpha_{k}^{(1)}=\alpha_{k}^{(2)}=\alpha_{k}$ for every $k>2$. Since $\alpha_{1}<2^{n / p}$, we can find small enough $\varepsilon>0$ such that $g_{i}$ satisfy $\left\|\left|g_{i}\right|\right\|_{p, \infty}^{[0,1]} \leq 1$, for $i=1,2$. Indeed, for $i=1$, we need to prove that for small enough $\varepsilon>0$

$$
\int_{0}^{t} g_{1} \leq t^{1-\frac{1}{p}}
$$

for every $t \in[0,1)$, since $g_{1}$ is decreasing. (3.2) is now obviously true for $t \geq \frac{2}{2^{n}}$ since

$$
\int_{0}^{t} g_{1}=\int_{0}^{t} g^{*} \text { for every such } t
$$

(3.2) is also true for $t=0, \frac{1}{2^{n}}$ for a suitable $\varepsilon>0$. But then it remains true for every $t \in\left(0, \frac{1}{2^{n}}\right)$ since the function $t \mapsto \int_{0}^{t} g_{1}$ represents a straight line on $\left[0, \frac{1}{2^{n}}\right]$ and $t^{1-\frac{1}{p}}$ is concave there, analogously for the interval $\left[\frac{1}{2^{n}}, \frac{2}{2^{n}}\right]$. That is we proved $\|\left.\left|g_{1}\right|\right|_{p, \infty} ^{[0,1]} \leq 1$. For $i=2$ we use the same arguments and the hypothesis $\alpha_{1}>\alpha_{2}$ in order to ensure that for small enough $\varepsilon>0, g_{2}$ is decreasing. Obviously now, 
$\int_{0}^{1} g_{i}=f$, so that $g_{i} \in \Delta_{n}$, for $i=1,2$. But $g^{*}=\frac{g_{1}+g_{2}}{2}$, with $g_{i} \neq g$ and $g_{i} \in \Delta_{n}$, $i=1,2$, , a contradiction since $g^{*} \in \operatorname{ext}\left(\Delta_{n}\right)$. So,

$$
\alpha_{1}=2^{n / p} \text { and } \quad \int_{0}^{1 / 2} g^{*}=\left(\frac{1}{2^{n}}\right)^{1-\frac{1}{p}}
$$

that is what we wanted to prove. In the same way we prove that for $i \in\{1,2, \ldots$, $\left.2^{n}-1\right\}$ such that

$$
\left(\frac{i+1}{2^{n}}\right)^{1-\frac{1}{p}} \leq f, \quad \text { if } \quad \int_{0}^{i / 2^{n}} g^{*}=\left(\frac{i}{2^{n}}\right)^{1-\frac{1}{p}}, \quad \text { then } \quad \int_{0}^{(i+1) / 2^{n}} g^{*}=\left(\frac{i+1}{2^{n}}\right)^{1-\frac{1}{p}} .
$$

The lemma is now proved by induction.

Let now $g \in \operatorname{ext}\left(\Delta_{n}\right)$ and $k=\max \left\{i \leq 2^{n}:\left(\frac{i}{2^{n}}\right)^{1-\frac{1}{p}} \leq f\right\}$, so if we suppose that $f<1$, we have that

$$
\left(\frac{k}{2^{n}}\right)^{1-\frac{1}{p}} \leq f<\left(\frac{k+1}{2^{n}}\right)^{1-\frac{1}{p}}
$$

By Lemma 3.2,

$$
\int_{0}^{k / 2^{n}} g^{*}=\left(\frac{k}{2^{n}}\right)^{1-\frac{1}{p}}
$$

But by using the reasoning of the previous lemma it is easy to see that

$$
\int_{0}^{(k+1) / 2^{n}} g^{*}=f
$$

which gives

$$
\int_{k / 2^{n}}^{k+1 / 2^{n}} g^{*}=f-\left(\frac{k}{2^{n}}\right)^{1-\frac{1}{p}} \Longrightarrow \alpha_{k+1}=2^{n} \cdot f-2^{n / p} \cdot k^{1-\frac{1}{p}} .
$$

Additionally, $\alpha_{i}=0$ for $i>k+1$. From the above we obtain the following

Corollary 3.1. Let $g \in \operatorname{ext}\left(\Delta_{n}\right)$. Then $g^{*}=\sum_{i=1}^{2^{n}} \alpha_{i} \xi_{I_{i}}$, where

$$
\alpha_{i}=2^{n / p}\left(i^{1-\frac{1}{p}}-(i-1)^{1-\frac{1}{p}}\right) \text { for } i=1,2, \ldots, k
$$

and

where

$$
\alpha_{k+1}=2^{n} f-2^{n / p} \cdot k^{1-\frac{1}{p}}, \quad \alpha_{i}=0, \quad i>k+1,
$$

$$
k=\max \left\{i \leq 2^{n}:\left(\frac{i}{2^{n}}\right)^{1-\frac{1}{p}} \leq f\right\} .
$$

We estimate now the $L^{q}$-norm of every $g \in \operatorname{ext}\left(\Delta_{n}\right)$. We state it as

Lemma 3.3. Let $g \in \operatorname{ext}\left(\Delta_{n}\right)$ and $A=\int_{0}^{1} g^{q}$. Then $A \leq \Gamma f^{p-q / p-1}+\mathcal{E}_{n}(f)$, where

$$
\Gamma=\left(\frac{p-1}{p}\right)^{q} \frac{p}{p-q} \quad \text { and } \quad \mathcal{E}_{n}(f)=\frac{\alpha_{k+1}^{q}}{2^{n}}=\frac{\left(2^{n} f-2^{n / p} k^{1-\frac{1}{p}}\right)^{q}}{2^{n}}
$$


Proof. For $g$ we write $g^{*}=\sum_{i=1}^{2^{n}} \alpha_{i} \xi_{I_{i}}$, where $\alpha_{i}$ are given in Corollary 3.1. Then

$$
A=\int_{0}^{1}\left(g^{*}\right)^{q}=\left[\left(\sum_{i=1}^{k} \alpha_{i}^{q}\right)+\alpha_{k+1}^{q}\right] \cdot \frac{1}{2^{n}} .
$$

Now for $i \in\{1,2, \ldots, k\}$

$$
\begin{aligned}
\alpha_{i}^{q} & =\left[2^{n / p}\left(i^{1-\frac{1}{p}}-(i-1)^{1-\frac{1}{p}}\right)\right]^{q}=\left\{2^{n}\left[\left(\frac{i}{2^{n}}\right)^{1-\frac{1}{p}}-\left(\frac{i-1}{2^{n}}\right)^{1-\frac{1}{p}}\right]\right\}^{q} \\
& =\left[2^{n} \int_{i-1 / 2^{n}}^{i / 2^{n}} \psi\right]^{q},
\end{aligned}
$$

where $\psi:(0,1] \rightarrow \mathbf{R}^{+}$is defined by $\psi(t)=\frac{p-1}{p} t^{-1 / p}$. By (3.5) and in view of Hölder's inequality we have that for $i \in\{1,2, \ldots, k\}$

$$
\alpha_{i}^{q} \leq 2^{n} \int_{i-1 / 2^{n}}^{i / 2^{n}} \psi^{q}
$$

Summing up relations (3.6) we have that

$$
\sum_{i=1}^{k} \alpha_{i}^{q} \leq 2^{n} \int_{0}^{k / 2^{n}} \psi^{q}=2^{n} \cdot \Gamma \cdot\left(\frac{k}{2^{n}}\right)^{1-\frac{q}{p}} .
$$

Additionally from the definition of $k$ we have that

$$
\left(\frac{k}{2^{n}}\right)^{1-\frac{1}{p}} \leq f \Longrightarrow k^{1-\frac{q}{p}} \leq\left(2^{n}\right)^{1-\frac{q}{p}} \cdot f^{p-q / p-1} .
$$

From (3.4), (3.7) and (3.8) we obtain

$$
A \leq\left[2^{n} \cdot \Gamma \cdot f^{p-q / p-1}+\alpha_{k+1}^{q}\right] \frac{1}{2^{n}}=\Gamma f^{p-q / p-1}+\mathcal{E}_{n}(f)
$$

and Lemma 3.3 is proved.

Corollary 3.2. For every $g \in \Delta_{n}$,

$$
A \leq \Gamma f^{p-q / p-1}+\mathcal{E}_{n}(f), \text { where } A=\int_{0}^{1} g^{q} .
$$

Proof. This is true, of course, for $g \in \operatorname{ext}\left(\Delta_{n}\right)$, and so also for $g \in \operatorname{conv}\left(\operatorname{ext} \Delta_{n}\right)$, since $t \mapsto t^{q}$ is convex for $q>1$ on $\mathbf{R}^{+}$. It remains true for $g \in \overline{\operatorname{conv}\left(\operatorname{ext}\left(\Delta_{n}\right)\right)} L^{p, \infty}([0,1])$ using a simple continuity argument. In fact, we just need the continuity of the identity operator if it is viewed as $I: L^{p, \infty}([0,1]) \rightarrow L^{q}([0,1])$. See [4]. Using now Krein-Milman Theorem the Corollary is proved.

We have now the following

Corollary 3.3. Let $\phi:(X, \mu) \rightarrow \mathbf{R}^{+}$such that

$$
\int_{X} \phi d \mu=f, \quad \int_{X} \phi^{q} d \mu=A, \quad\|\phi\|_{p, \infty} \leq 1
$$

Then

$$
f^{q} \leq A \leq \Gamma f^{p-q / p-1}
$$


Proof. Let $g=\phi^{*}:[0,1] \rightarrow \mathbf{R}^{+}$. There exist a sequence $\left(g_{n}\right)$ of $\frac{1}{2^{n}}$-simple functions, such that $g_{n} \leq g_{n+1} \leq g$ and $g_{n}$ converges almost everywhere to $g$. But then by defining

we have that

$$
f_{n}=\int_{0}^{1} g_{n}, \quad A_{n}=\int_{0}^{1} g_{n}^{q}
$$

$$
g_{n} \in \Delta_{n}\left(f_{n}\right) \text { so that } A_{n} \leq \Gamma f_{n}^{p-q / p-1}+\mathcal{E}_{n}\left(f_{n}\right) .
$$

By the monotone convergence theorem $f_{n} \rightarrow f, A_{n} \rightarrow A$. Moreover,

$$
\mathcal{E}_{n}\left(f_{n}\right)=\frac{\left(2^{n} f_{n}-k_{n}^{1-\frac{1}{p}} 2^{n / p}\right)^{q}}{2^{n}}
$$

where $k_{n}$ satisfy

$$
\left(\frac{k_{n}}{2^{n}}\right)^{1-\frac{q}{p}} \leq f_{n}<\left(\frac{k_{n}+1}{2^{n}}\right)^{1-\frac{1}{p}}
$$

As a consequence

$$
\begin{aligned}
\mathcal{E}_{n}\left(f_{n}\right) & =\left(2^{n}\right)^{q-1}\left[f_{n}-\left(\frac{k_{n}}{2^{n}}\right)^{1-\frac{1}{p}}\right]^{q}<\left(2^{n}\right)^{q-1}\left[\left(\frac{k_{n}+1}{2^{n}}\right)^{1-\frac{1}{p}}-\left(\frac{k_{n}}{2^{n}}\right)^{1-\frac{1}{p}}\right]^{q} \\
& \leq\left(2^{n}\right)^{q-1}\left[\left(\frac{1}{2^{n}}\right)^{1-\frac{1}{q}}\right]^{q}=\left(\frac{1}{2^{1-\frac{q}{p}}}\right)^{n} \rightarrow 0, \text { as } n \rightarrow \infty
\end{aligned}
$$

where in the second inequality we used the known

$$
(t+s)^{\alpha} \leq t^{\alpha}+s^{\alpha} \quad \text { for } t, s \geq 0,0<\alpha<1 .
$$

Now (3.9) gives the corollary.

In fact the converse of Corollary 3.3 is also true.

Theorem 3.2. For $0<f \leq 1, A>0$ the following are equivalent:

i) $f^{q} \leq A \leq \Gamma f^{p-q / p-1}$,

ii) $\exists \phi:(X, \mu) \rightarrow \mathbf{R}^{+}$such that

$$
\int_{X} \phi d \mu=f, \quad \int_{X} \phi^{q} d \mu=A, \quad\|\phi \mid\|_{p, \infty} \leq 1 .
$$

We prove first the following

Lemma 3.4. Let $\alpha \in(0,1)$ and $(f, A)$ such that

$$
\begin{aligned}
f & \lesseqgtr \alpha^{1-\frac{1}{p}}, \\
f^{q} & \lesseqgtr \alpha^{q-1} A, \\
A & \leq \Gamma f^{p-q / p-1} .
\end{aligned}
$$

Then there exists $g:[0, \alpha] \rightarrow \mathbf{R}^{+}$such that

$$
\int_{0}^{\alpha} g=f, \quad \int_{0}^{\alpha} g^{q}=A, \quad \text { and } \quad\|g\|_{p, \infty}^{[0, \alpha]}=1,
$$

where

$$
\|\mid\| \|_{p, \infty}^{[0, \alpha]}=\sup \left\{|E|^{-1+\frac{1}{p}} \int_{E} g: E \text { measurable subset of }[0, \alpha] \text { such that }|E|>0\right\} .
$$


Proof. We search for a $g$ of the form

$$
g:= \begin{cases}\frac{p-1}{p} t^{-1 / p}, & 0<t \leq c_{1} \\ \mu_{2}, & c_{1}<t \leq \alpha\end{cases}
$$

for suitable constant $c_{1} \mu_{2}$. We must have that

$$
\int_{0}^{\alpha} g=f \Longleftrightarrow c_{1}^{1-\frac{1}{p}}+\mu_{2}\left(\alpha-c_{1}\right)=f .
$$

Additionally, $g$ must satisfy

$$
\int_{0}^{\alpha} g^{q}=A \Longleftrightarrow \Gamma c_{1}^{1-\frac{q}{p}}+\mu_{2}^{q}\left(\alpha-c_{1}\right)=A .
$$

(3.13) gives

$$
\mu_{2}=\frac{f-c_{1}^{1-\frac{1}{p}}}{a-c_{1}}
$$

so (3.14) becomes

$$
\Gamma c_{1}^{1-\frac{q}{p}}+\frac{\left(f-c_{1}^{1-\frac{1}{P}}\right)^{q}}{\left(\alpha-c_{1}\right)^{q-1}}=A .
$$

That is we search for a $c_{1} \in(0, \alpha)$ such that

$$
T\left(c_{1}\right)=A \text { where } T:[0, \alpha) \rightarrow \mathbf{R}^{+}
$$

is defined by

$$
T(t)=\Gamma t^{1-\frac{q}{p}}+\frac{\left(f-t^{1-\frac{1}{p}}\right)^{q}}{(\alpha-t)^{q-1}} .
$$

Observe that $T(0)=\frac{f^{q}}{\alpha^{q-1}} \lesseqgtr A$ because of $(3.11)$ and that $T\left(f^{p / p-1}\right)=\Gamma f^{p-q / p-1} \geq$ $A$. Now because of the continuity of $T$, there exists $c_{1} \in\left(0, f^{p / p-1}\right]$ such that $T\left(c_{1}\right)=$ $A$. Then $c_{1} \in(0, \alpha)$ because of $(3.10)$, and if we define $\mu_{2}$ by $(3.15)$, we guarantee (3.13) and (3.14). We need to prove now that $\||| g\|_{p, \infty}^{[0, \alpha]}=1$. Obviously, because of the form of $g,\|\mid\| g \|_{p, \infty}^{[0, \alpha]} \geq 1$. So we have to prove that

$$
\int_{0}^{t} g \leq t^{1-\frac{1}{p}}, \quad \forall t \in(0, \alpha] .
$$

This is of course true for $t \in\left[0, c_{1}\right]$. For $t \in\left(c_{1}, \alpha\right]$,

$$
\int_{0}^{t} g=c_{1}^{1-\frac{1}{p}}+\mu_{2}\left(t-c_{1}\right)=: G(t)
$$

Since $G\left(c_{1}\right)=c_{1}^{1-\frac{1}{p}}, G(\alpha)=f<\alpha^{1-\frac{1}{p}}$ and $t \mapsto t^{1-\frac{1}{p}}$ is concave on $\left(c_{1}, \alpha\right]$, (3.17) is true. Thus Lemma 3.4 is proved.

We have now the

Proof of Theorem 3.2. We have to prove the direction i) $\Rightarrow$ ii). Indeed, if $f^{q} \lesseqgtr A \leq \Gamma f^{p-q / p-1}$ and $f<1$, we apply Lemma 3.4. If $f^{q}=A$ with $0<f \leq 1$, we set $g$ by $g(t)=f$, for every $t \in[0,1]$, while if $f=1 \leq A \leq \Gamma$ a simple modification of Lemma 3.4 gives the result. 
We conclude Section 3 with the following theorem which can be proved easily using all the above.

Theorem 3.3. For $f, A$ such that $0<f<1, A>0$ the following are equivalent:

i) $f^{q} \lesseqgtr A \leq \Gamma f^{p-q / p-1}$,

ii) $\exists \phi:(X, \mu) \rightarrow \mathbf{R}^{+}$such that $\int_{X} \phi d \mu=f, \quad \int_{X} \phi^{q} d \mu=A, \quad\|\phi \mid\|_{p, \infty}=1$.

Remark 3.1. Theorem 3.3 is completed if we mention that for $f=1$ the following are equivalent:

i) $f=1 \leq A \leq \Gamma$,

ii) $\exists \phi:(X, \mu) \rightarrow \mathbf{R}^{+}$such that $\int_{X} \phi d \mu=1, \quad \int_{X} \phi^{q} d \mu=A, \quad\||\phi|\|_{p, \infty}=1$.

\section{The extremal problem}

Let $\mathcal{M}_{\mathcal{T}}=\mathcal{M}$ the dyadic maximal operator associated to the tree $\mathcal{T}$, on the probability non-atomic measure space $(X, \mu)$. Our aim is to find

$$
T_{f, A, F}(\lambda)=\sup \left\{\mu(\{\mathcal{M} \phi \geq \lambda\}): \phi \geq 0, \int_{X} \phi d \mu=f, \int_{X} \phi^{q} d \mu=A,\|\| \|_{p_{, \infty}}=F\right\}
$$

for all the allowable values of $f, A, F$. We find it in the case where $F=1$. We write $T_{f, A}(\lambda)$ for $T_{f, A, 1}(\lambda)$. In order to find $T_{f, A}(\lambda)$ we find first the following

$$
T_{f, A}^{(1)}(\lambda)=\sup \left\{\mu(\{\mathcal{M} \phi \geq \lambda\}): \phi \geq 0, \int_{X} \phi d \mu=f, \int_{X} \phi^{q} d \mu=A,\|\phi\|_{p, \infty} \leq 1\right\} .
$$

The domain of this extremal problem is the following

$$
D=\left\{(f, A): 0<f \leq 1, f^{q} \leq A \leq \Gamma f^{p-q / p-1}\right\} .
$$

Obviously, $T_{f, A}^{(1)}(\lambda)=1$, for $\lambda \leq f$. Let now $\lambda>f$ and $(f, A) \in D$. Let $\phi$ be as in the definition of $T_{f, A}^{(1)}(\lambda)$. Consider the decreasing rearrangement of $\phi, g=\phi^{*}:[0,1] \rightarrow$ $\mathbf{R}^{+}$. Then

$$
\int_{0}^{1} g=f, \quad \int_{0}^{1} g^{q}=A, \quad\|g\|_{p, \infty}^{[0,1]} \leq 1 .
$$

Consider also $E=\{\mathcal{M} \phi \geq \lambda\} \subseteq X$. Then $E$ is the almost disjoint union of elements of $\mathcal{T}$, let $\left(I_{j}\right)_{j}$. In fact, we just need to consider the elements $I$ of $\mathcal{T}$, maximal under the condition

$$
\frac{1}{\mu(I)} \int_{I} \phi d \mu \geq \lambda
$$

We then have $E=\bigcup_{j} I_{j}$ and $\int_{E} \phi d \mu \geq \lambda \mu(E)$ because of (4.1). Then according to Lemma 2.1 we have that $\int_{0}^{\alpha} g \geq \alpha \lambda$ where $\alpha=\mu(E)$. That is

$$
T_{f, A}^{(1)}(\lambda) \leq \Delta_{f, A}(\lambda)
$$

where

$$
\begin{aligned}
\Delta_{f, A}(\lambda)=\sup \left\{\alpha \in(0,1]: \exists g:[0,1] \rightarrow \mathbf{R}^{+}:\right. \\
\\
\left.\int_{0}^{1} g=f, \int_{0}^{1} g^{q}=A,\|\| g \|_{p, \infty}^{[0,1]} \leq 1, \int_{0}^{\alpha} g \geq \alpha \lambda\right\} .
\end{aligned}
$$


We prove now the converse inequality in (4.2) by proving the following

Lemma 4.1. Let $g$ be as in (4.3) for a fixed $\alpha \in(0,1]$. Then there exists $\phi:(X, \mu) \rightarrow \mathbf{R}^{+}$such that

$$
\int_{X} \phi d \mu=f, \quad \int_{X} \phi^{q} d \mu=A, \quad\||\phi|\|_{p, \infty} \leq 1 \text { and } \mu(\{\mathcal{M} \phi \geq \lambda\}) \geq \alpha .
$$

Proof. Lemma 2.3 guarantees the existence of a sequence $\left(I_{j}\right)_{j}$ of pairwise almost disjoint elements of $\mathcal{T}$ such that

$$
\mu\left(\bigcup I_{j}\right)=\sum \mu\left(I_{j}\right)=\alpha .
$$

Consider now the finite measure space $([0, \alpha],|\cdot|)$, where $|\cdot|$ is the Lebesque measure. Then since $\int_{0}^{\alpha} g \geq \alpha \lambda$ and (4.4) holds, applying Lemma 2.2 repeatedly, we obtain the existence of a sequence $\left(A_{j}\right)$ of Lebesque measurable subsets of $[0, \alpha]$ such that the following hold:

$\left(A_{j}\right)_{j}$ is a pairwise disjoint family, $\bigcup A_{j}=[0, \alpha],\left|A_{j}\right|=\mu\left(I_{j}\right), \frac{1}{\left|A_{j}\right|} \int_{A_{j}} g \geq \lambda$.

Then we define $g_{j}:\left[0,\left|A_{j}\right|\right] \rightarrow \mathbf{R}^{+}$by $g_{j}=\left(g / A_{j}\right)^{*}$. Define also for every $j$ a measurable function $\phi_{j}: I_{j} \rightarrow \mathbf{R}^{+}$so that $\phi_{j}^{*}=g_{j}$. The existence of such a function is guaranteed by the fact that $\left(I_{j}, \mu / I_{j}\right)$ is non-atomic. Since $\left(I_{j}\right)$ is almost pairwise disjoint family we produce a $\phi^{(1)}: \cup I_{j} \rightarrow \mathbf{R}^{+}$measurable such that $\phi^{(1)} / I_{j}=\phi_{j}$. We set now $Y=X \backslash \cup I_{j}$ and $h:[0,1-\alpha] \rightarrow \mathbf{R}^{+}$by $h=(g /[\alpha, 1])^{*}$. Then since $\mu(Y)=1-\alpha$ there exists $\phi^{(2)}: Y \rightarrow \mathbf{R}^{+}$such that $\left(\phi^{(2)}\right)^{*}=h$. Set now

$$
\phi= \begin{cases}\phi^{(1)}, & \text { on } \cup I_{j} \\ \phi^{(2)}, & \text { on } Y\end{cases}
$$

It is easy to see from the above construction that $\phi^{*}=g$ a.e. with respect to Lesbesgue measure, which gives $\int_{X} \phi d \mu=f, \int_{X} \phi^{q} d \mu=A$ and \|\|$\phi \|_{p, \infty} \leq 1$. Additionally,

$$
\frac{1}{\mu\left(I_{j}\right)} \int_{I_{j}} \phi d \mu=\frac{1}{\left|A_{j}\right|} \int_{A_{j}} g \geq \lambda \quad \text { for every } j
$$

that is,

$$
\{\mathcal{M} \phi \geq \lambda\} \supseteq \cup I_{j}, \quad \text { so } \mu(\{\mathcal{M} \phi \geq \lambda\}) \geq \alpha
$$

and the lemma is proved.

It is now not difficult to see that we can replace the inequality $\int_{0}^{\alpha} g \geq \alpha \lambda$ in the definition of $\Delta_{f, A}(\lambda)$ by equality, thus defining $S_{f, A}(\lambda)$, in such a way that

$$
T_{f, A}^{(1)}(\lambda)=\Delta_{f, A}(\lambda)=S_{f, A}(\lambda)
$$

This is true since if $g$ is as in (4.3) and $\lambda>f$, there exists $\beta \geq \alpha$ such that $\int_{0}^{\beta} g=\beta \lambda$. For $(f, A) \in D$ we set

$$
G_{f, A}(\lambda)=\sup \left\{\mu(\{\mathcal{M} \phi \geq \lambda\}): \phi \geq 0, \int_{X} \phi d \mu=f, \int_{X} \phi^{q} d \mu=A\right\} .
$$

It is obvious that $T_{f, A}^{(1)}(\lambda) \leq G_{f, A}(\lambda)$. As a matter of fact $G_{f, A}(\lambda)$ has been computed in [6] and was found to be 


$$
G_{f, A}(\lambda)= \begin{cases}1, & \lambda \leq f, \\ \frac{f}{\lambda}, & f<\lambda<\left(\frac{A}{f}\right)^{1 / q-1} \\ k, & \left(\frac{A}{f}\right)^{1 / q-1} \leq \lambda,\end{cases}
$$

where $k$ is the unique root of the equation

$$
\frac{(f-\alpha \lambda)^{q}}{(1-\alpha)^{q-1}}+\alpha \lambda^{q}=A \text { on } \alpha \in\left[0, \frac{f}{\lambda}\right] \text {, when } \lambda>\left(\frac{A}{f}\right)^{1 / q-1} .
$$

We have now the following

Proposition 4.1. If $(f, A) \in D$, then

$$
T_{f, A}^{(1)}(\lambda) \leq \min \left\{1, G_{f, A}(\lambda), \frac{1}{\lambda^{p}}\right\} .
$$

Proof. We just need to see that $\mu(\{\mathcal{M} \phi \geq \lambda\}) \leq \frac{1}{\lambda^{p}}$ for every $\phi$ such that $\||| \phi \mid\|_{p, \infty} \leq 1$. But if $E=\{\mathcal{M} \phi \geq \lambda\}$, we have by the definition of the norm \|\|$\cdot\|\|_{p, \infty}$ that $\int_{E} \phi \leq \mu(E)^{1-\frac{1}{p}}$. But by (1.3) $\int_{E} \phi \geq \lambda \mu(E)$, so that

$$
\lambda \mu(E) \leq \mu(E)^{1-\frac{1}{p}} \Longrightarrow \mu(E) \leq \frac{1}{\lambda^{p}} .
$$

So Proposition 4.1 is true.

We prove now that in Proposition 4.1 we have equality.

Proposition 4.2. Let $(f, A) \in D$ and $\lambda$ such that

$$
\frac{f}{\lambda}=\min \left\{1, G_{f, A}(\lambda), \frac{1}{\lambda^{p}}\right\}
$$

Then $T_{f, A}^{(1)}(\lambda)=\frac{f}{\lambda}$.

Proof. We use Lemma 3.4 and equations (4.5). Because of (4.5) we need to find $g:[0,1] \rightarrow \mathbf{R}^{+}$such that

$$
\int_{0}^{1} g=f, \quad \int_{0}^{1} g^{q}=A, \quad\|g\|_{p, \infty} \leq 1 \quad \text { and } \quad \int_{0}^{f / \lambda} g=\frac{f}{\lambda} \cdot \lambda=f,
$$

that is, $g$ should be defined on $[0, f / \lambda]$. We apply Lemma 3.4, with $\alpha=\frac{f}{\lambda}$. In fact, since (4.7) is true, we have that $G_{f, A}(\lambda)=\frac{f}{\lambda}$ so, $\lambda<\left(\frac{A}{f}\right)^{1 / q-1}$ which gives (3.11), while $\frac{f}{\lambda} \leq \frac{1}{\lambda^{p}}$ gives (3.10). In fact, Lemma 3.4 works even with equality on (3.10) as it is easily can be seen by continouity reasons. So, in view of (4.5) we have $T_{f, A}^{(1)}(\lambda) \geq f / \lambda$ and the proposition is proved.

At the next step we have

Proposition 4.3. Let $(f, A) \in D$ and $\lambda$ such that

$$
k=\min \left\{1, G_{f, A}(\lambda) \frac{1}{\lambda^{p}}\right\} .
$$

Then $T_{f, A}^{(1)}(\lambda)=k$. 
Proof. Obviously, (4.8) gives $\lambda \geq\left(\frac{A}{f}\right)^{1 / q-1}$. We prove that there exists $g:[0,1] \rightarrow$ $\mathbf{R}^{+}$such that

$$
\int_{0}^{k} g=k \lambda, \quad \int_{0}^{1} g=f, \quad \int_{0}^{1} g^{q}=A \text { and } \quad\|g\|_{p, \infty} \leq 1 .
$$

For this purpose we define

$$
g:= \begin{cases}\lambda, & \text { on }[0, k], \\ \frac{f-k \lambda}{1-k}, & \text { on }(k, 1] .\end{cases}
$$

Then, obviously, the first two conditions in (4.9) are satisfied, while

$$
\int_{0}^{1} g^{q}=\frac{(f-k \lambda)^{q}}{(1-k)^{q-1}}+k \lambda^{q}=A
$$

by the definition of $k$. Moreover, $\||| g \mid\|_{p, \infty} \leq 1$. This is true since $k \lambda \leq k^{1-\frac{q}{p}}, f \leq 1$ and the fact that $g$ is constant on each of the intervals $[0, k]$ and $(k, 1]$. So the proposition is proved.

At last we prove

Proposition 4.4. Let $(f, A) \in D$ and $\lambda$ such that

$$
\frac{1}{\lambda^{p}}=\min \left\{1, G_{f, A}(\lambda), \frac{1}{\lambda^{p}}\right\} .
$$

Then $T_{f, A}^{(1)}(\lambda)=\frac{1}{\lambda^{p}}$.

Proof. As before we search for a function $g$ such that

$$
\int_{0}^{1} g=f, \quad \int_{0}^{1} g^{q}=A, \quad\|g\|_{p, \infty} \leq 1 \quad \text { and } \quad \int_{0}^{1 / \lambda^{p}} g=\frac{1}{\lambda^{p}} \cdot \lambda=\frac{1}{\lambda^{p-1}} .
$$

We define

$$
\vartheta_{\lambda}=\frac{\Gamma}{\lambda^{p-q}}+\frac{\left(f-\frac{1}{\lambda^{p-1}}\right)^{q}}{\left(1-\frac{1}{\lambda^{p}}\right)^{q-1}}
$$

and we consider two cases:

i) $\vartheta_{\lambda}>A$. We search for a function of the form

$$
g:= \begin{cases}\left(1-\frac{1}{p}\right) t^{-1 / p}, & 0<t \leq c_{1}, \\ \mu_{2}, & c_{1}<t \leq \frac{1}{\lambda^{p}}, \\ \mu_{3}, & \frac{1}{\lambda^{p}}<t<1,\end{cases}
$$

for suitable constants $c_{1} \leq \frac{1}{\lambda^{p}}, \mu_{2}, \mu_{3}$. Then in view of (4.11) the following must hold:

$$
\begin{aligned}
c_{1}^{1-\frac{1}{p}}+\mu_{2}\left(\frac{1}{\lambda^{p}}-c_{1}\right) & =\frac{1}{\lambda^{p-1}}, \\
c_{1}^{1-\frac{1}{p}}+\mu_{2}\left(\frac{1}{\lambda^{p}}-c_{1}\right)+\mu_{3}\left(1-\frac{1}{\lambda^{p}}\right) & =f, \\
\Gamma c_{1}^{1-\frac{q}{p}}+\mu_{2}^{q}\left(\frac{1}{\lambda^{p}}-c_{1}\right)+\mu_{3}^{q}\left(1-\frac{1}{\lambda^{p}}\right) & =A .
\end{aligned}
$$


Notice that the condition $\||g|\|_{p, \infty} \leq 1$ is automatically satisfied because of the form of $g$ and the previous stated relations. Now (4.13) and (4.14) give

$$
\mu_{3}=\frac{f-\frac{1}{\lambda^{p-1}}}{1-\frac{1}{\lambda^{p}}}
$$

and

$$
\mu_{2}=\frac{\frac{1}{\lambda^{p-1}}-c_{1}^{1-\frac{1}{p}}}{\frac{1}{\lambda^{p}}-c_{1}}
$$

while (4.15) gives $T\left(c_{1}\right)=A$ where $T$ is defined on $\left[0, \frac{1}{\lambda^{p}}\right)$ by

$$
T(c)=\Gamma c^{1-\frac{q}{p}}+\frac{\left(\frac{1}{\lambda^{p-1}}-c^{1-\frac{1}{p}}\right)^{q}}{\left(\frac{1}{\lambda^{p}}-c\right)^{q-1}}+\frac{\left(f-\frac{1}{\lambda^{p-1}}\right)^{q}}{\left(1-\frac{1}{\lambda^{p}}\right)^{q-1}} .
$$

Then

$$
T(0)=\frac{1}{\lambda^{p-q}}+\frac{\left(f-\frac{1}{\lambda^{p-1}}\right)^{q}}{\left(1-\frac{1}{\lambda^{p}}\right)^{q-1}} .
$$

It is now easy to see that $T(0) \leq A$ by using that $F:[0, f / \lambda] \rightarrow \mathbf{R}^{+}$defined by

$$
F(t)=\frac{(f-t \lambda)^{q}}{(1-t)^{q-1}}+t \lambda^{q}
$$

is increasing, and the definition of $G_{f, A}(\lambda)$. Moreover $\lim _{c \rightarrow \frac{1-}{\lambda^{p}}} T(c)=\vartheta_{\lambda}>A$, so by continuity of the function $t$, we end case i). Now for

ii) $\vartheta_{\lambda} \leq A$. We search for a function of the form

$$
g:= \begin{cases}\left(1-\frac{1}{p}\right) t^{1-1 / p}, & 0<t \leq c_{1}, \\ \mu_{2}, & c_{1}<t \leq 1\end{cases}
$$

where $\frac{1}{\lambda^{p}}<c_{1}$. Similar arguments as in case i) give the result.

From Propositions 4.1-4.4 we have now

Theorem 4.1. For $(f, A) \in D$,

$$
T_{f, A}^{(1)}(\lambda)=\min \left\{1, G_{f, A}(\lambda), \frac{1}{\lambda^{p}}\right\} .
$$

Remark 4.1. Notice that $T_{f, A}(\lambda)=T_{f, A}^{(1)}(\lambda)$ for every $f, A$ such that $f^{q}<A \leq$ $\Gamma f^{p-q / p-1}$ and $0<f \leq 1$. Indeed, suppose that $\alpha=T_{f, A}^{(1)}(\lambda)$. Then there exists $g:[0,1] \rightarrow \mathbf{R}^{+}$such that

$$
\int_{0}^{1} g=f, \quad \int_{0}^{1} g^{q}=A, \quad \int_{0}^{\alpha} g=\alpha \lambda \text { and }\|g\|_{p, \infty} \leq 1 .
$$

It is easy to see that for every $\varepsilon>0$, small enough we can produce from $g$ a function $g_{\varepsilon}$ satisfying

$$
\int_{0}^{\alpha-\varepsilon} g_{\varepsilon} \geq(\alpha-\varepsilon) \lambda, \quad \int_{0}^{1} g_{\varepsilon}=f, \quad \int_{0}^{1} g_{\varepsilon}=A+\delta_{\varepsilon} \quad \text { and } \quad\|\| g_{\varepsilon} \|\left.\right|_{p, \infty}=1,
$$


where $\lim _{\varepsilon \rightarrow 0^{+}} \delta \varepsilon=0$. This and continuity reasons shows $T_{f, A}(\lambda)=\alpha$.

iii) The case $A=f^{q}$ can be worked out separately because there is essentially unique function $g$ satisfying $\int_{0}^{1} g=f, \int_{0}^{1} g^{q}=f^{q}$, namely the constant function with value $f$.

Scaling all the above we have that

Theorem 4.2. For $f$, $A$ such that $f^{q}<A \leq \Gamma f^{p-q / p-1} F^{p(q-1) /(p-1)}$ and $0<$ $f \leq F$ the following hold

$$
\begin{aligned}
& \sup \left\{\mu(\{\mathcal{M} \phi \geq \lambda\}): \phi \geq 0, \int_{X} \phi d \mu=f, \int_{X} \phi^{q} d \mu=A,\|\mid \phi\|_{p, \infty}=F\right\} \\
& =\min \left\{1, G_{f, A}(\lambda), \frac{F^{p}}{\lambda^{p}}\right\}
\end{aligned}
$$

and

$$
\sup \left\{\|\mathcal{M} \phi\|_{p, \infty}: \phi \geq 0, \int_{X} \phi d \mu=f, \int_{X} \phi^{q} d \mu=A,\|\| \phi \mid \|_{p, \infty}=F\right\}=F .
$$

\section{References}

[1] Bennet, C., and R. Sharpley: Interpolation of operators. - Academic Press.

[2] Burkholder, D. L.: Martingales and Fourier analysis in Banach spaces. - In: C.I.M.E. Lectures (Varenna (Como), Italy, 1985), Lecture Notes in Math. 1206, 1986, 61-108.

[3] Burkholder, D. L.: Boundary value problems and sharp inequalities for martingale transforms. - Ann. Probab. 12, 1984, 647-702.

[4] Grafakos, L.: Classical and modern Fourier analysis. - Pearson Education, Upper Saddle River, N.J., 2004.

[5] Melas, A. D.: The Bellman functions of dyadic-like maximal operators and related inequalities. - Adv. Math. 192, 2005, 310-340.

[6] Melas, A. D., and E. Nikolidakis: On weak type inequalities for dyadic maximal functions. - J. Math. Anal. Appl. 348, 2008, 404-410.

[7] Melas, A. D., and E. Nikolidakis: Dyadic-like maximal operators on integrable functions and Bellman functions related to Kolmogorov's inequality. - Trans. Amer. Math. Soc. 362:3, 1571-1596.

[8] Nazarov, F., and S. Treil: The hunt for a Bellman function: applications to estimates for singular integral operators and to other classical problems of harmonic analysis. - Algebra i Analiz 8:5, 1996, 32-162.

[9] Nikolidakis, E. N.: Extremal problems related to maximal dyadic-like operators. - J. Math. Anal. Appl. 369, 2010, 377-385.

[10] WANG, G.: Sharp maximal inequalities for conditionally symmetric martingales and Brownian motion. - Proc. Amer. Math. Soc. 112, 1991, 579-586. 